\title{
Dengue Outbreak and Management of Control Measures in the National Hospital of Sri Lanka
}

\author{
Dr. Sujatha Senaratne*
}

Dengue is a major public health problem in Sri Lanka. Globally, dengue is endemic in 112 countries. In Sri Lanka, in the year 2009 a major dengue epidemic with more than 334 known deaths and 34,659 clinically/laboratory confirmed cases has occurred, and also in the National Hospital of Sri Lanka (NHSL) there were hospital acquired confirmed dengue cases (37 staff members, and 21 in-ward patients admitted for other problems), and deaths (two inward patients, and two staff members) occurred during this epidemic. The NHSL is a 3000 -bed premier teaching hospital and the tertiary care facility and providing a wide spectrum of services. It is located on a 30 -acre block of land; employees of nearly 7000 including 100 consultant staff and over 800 doctors.

\section{Dengue Vector}

Primary dengue vector in Sri Lanka is Aedes aegypti, and secondary vector is Aedes albopictus. These vectors can breed even in a small quantity of water, and their eggs stick to the walls of the container at the surface of the water. Therefore, eggs may not be removed even if the water gets removed. Even if the water gets dried, Aedes eggs can survive and may hatch even after a one year when water gets collected again. Normally Aedes mosquitoes rest in the dark places especially bushes.

\section{Peak biting hours of Aedes Vectors}

Peak biting hours of the Aedes vectors 6.00 am to 10.00 am, and $4.00 \mathrm{pm}$ to $6.00 \mathrm{pm}$. Capable of biting several people in a short period for one blood meal, and the short flight range is $<200$ meters. As Aedes is a day biter a usual mosquito net does not give a protection for indoor patients to avoid dengue.

\section{Prevention of Dengue}

In a tropical country, it is not possible to completely eliminate the breeding or completely eradicate dengue when it becomes epidemics. The diseases could be controlled and kept at levels, so that they will not cause epidemics and become public health problems.

\section{Activities performed to control the spread of dengue in} the National Hospital premises:

\section{Epidemiological surveillance}

Epidemiological surveillance of dengue / DHF covers both disease and entomological surveillance. It is essential for monitoring endemic transmission and for early recognition of impending epidemics. Surveillance of dengue vectors is important in determining the distribution, major larval habits, spatial and temporal risk factors related to dengue transmission and level of insecticide susceptibility or resistance 1 . Therefore, a survey was conducted in the hospital with the assistance of the chief Epidemiologist, and Entomological staff of Medical Research Institute.

It was observed that Ae. aegypti and Ae. albopictus were breeding in a wide variety of natural and man-made containers (Brito Index $>25$ found most of the areas). The most preferred breeding sites of these vectors were blocked or broken drainages, tyres, refrigerator trays, metal ware, discarded containers, and roof gutters. Similarly, the Sri Lankan studies showed that ground level water storage tanks and barrels are the most productive breeding sites of Ae. aegypti and Ae. Albopictus 2, and it was reported that Thailand 3 had water storage tanks as the major breeding places of Aedes aegypti and Aedes albopictus.

\section{Hospital Dengue Prevention Committee}

New Hospital Dengue Prevention Committee was formed in NHSL. This committee conducted regular meetings, did regular weekly environmental inspection and took necessary steps for elimination of breeding sites and vector control activities for dengue prevention.

The committee adopted the following basic principles to control the hospital acquired dengue epidemics:

a. Reduce the buildup of mosquito densities,

b. Reduce the survival rate or the longevity of the mosquito.

c. Reduce or prevent man mosquito contact.

d. Identify and manage cases early.

Conducted Dengue death review meetings, and identified short comings which were discussed with the consultants. Also Public Health Inspectors of NHSL did regular inspections to identify breeding sites and reported to Dengue Prevention Committee for corrective action.

\section{Training of Hospital Staff}

Training program was organized for Medical officers, and other hospital staff. The aim of the program was to improve knowledge, attitude, practices and beliefs (KAP) of the hospital community on dengue prevention and control intervention. 


\section{Vector Control Activities}

Continuous Shramadana programs were conducted by junior health staff and cleaning staff to control vector breeding. These programs were conducted two days per week at every unit of the hospital, and they sent progress reports to the Infection Control Unit.

Weekly cleaning of water containers in all areas in the hospital premises, which is very effective for larval control. This was experienced in Fuji 4 and India 5. Also fixed tight lids to water storage tanks; weekly cleaning of roof gutters; added salt to refrigerator trays; filled earth pipes with sand; and filled water collecting areas in trees.

Space Spraying (Fogging) was done to kill the virus infected dengue vector mosquitoes to interrupt the transmission cycle of dengue, and the reduction of adult vector population.

All fever patients were provided with impregnated bed nets to protect other patients from mosquito borne diseases.

\section{Obstacles faced in carrying out activities}

Some of the drains outside NHSL premises were blocked, and they provide ideal breeding grounds for mosquitoes. Most of the street vendors along the Regent Street were disposing their garbage by throwing over the parapet wall into the NHSL premises as there was no proper garbage disposal system for them.

\section{Conclusion}

In this hospital the epidemic was controlled (B.I. was $<10$ to 0 in most of the breeding areas, and no new cases were reported) with hospital community participation and relevant inter and intra - sectoral collaboration. For any mosquito borne disease control programme to be successful, it should be well managed and be accepted by the community. Also, it should be sustainable and continue with full participation of the community.

\section{Reference}

1. World Health Organization. Guidelines for dengue surveillance and Mosquito Control. Western Pacific Education in Action Series No. 8. World Health Organisation Regional Office, Western Pacific, Manila, 1995.

2. Yapabandara, A.M.G.M., Abeykoon, A.R. Aedes aegypti and Aedes albopictus breeding sites in the Matale Municipal Council area. Proceedings of the Sri Lanka Association of the Advancement of Science (Abstract). 2002.

3. Phuanukoonnon, S., Mueller, I., Bryan, H. Effectiveness of dengue control practices in households water containers in Northeast Thailand. Trop. Med. And

Int. He. 2005; 10(8): 755-763.

4. Raju, A.K. Community Mobilization in Aedes aegypti control programme by source reduction in Peri-Urban District of Lautoka, Viti Levu, Fiji Islands Dengue Bulletin, 2003;27:149-155

5. Bohra, A. and Andrianasolo, H. Application of GIS modeling of dengue risk based on sociocultural Data: Case of Jalore, Rajastan, India. Dengue Bulletin, 2001;25:92-102 